\title{
Intensive vs Standard Blood Pressure Control in Adults 80 Years or Older: A Secondary Analysis of the Systolic Blood Pressure Intervention Trial
}

\author{
Nicholas M. Pajewski, PhD`, Dan R. Berlowitz, MD, MPH ${ }^{\dagger}$, , Adam P. Bress, PharmD $\S$, \\ Kathryn E. Callahan, MD", Alfred K. Cheung, MD**, Larry J. Fine, MD††, Sarah A. Gaussoin, \\ $M^{*}$, Karen C. Johnson, MD, MPH $\ddagger$, Jordan King, PharmD§,, , Dalane W. Kitzman, MD§§, \\ John B. Kostis, MD $\uparrow$ I, Alan J. Lerner, MD"\|", Cora E. Lewis, MD, MSPH ${ }^{\star \star *}$, Suzanne Oparil,

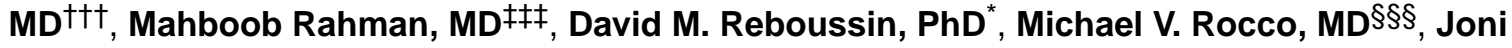

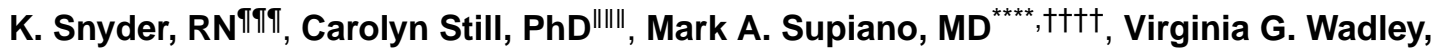 \\ PhD ${ }^{\dagger \dagger}$, Paul K. Whelton, MD ${ }^{\ddagger \ddagger \ddagger}$, Jackson T. Wright Jr, MD, PhD§§§§, Jeff D. Williamson, \\ MD, MHS"
}

*Department of Biostatistics and Data Science, Wake Forest School of Medicine, Winston-Salem, North Carolina; †Bedford Veterans Affairs Hospital, Bedford, Massachusetts; ‡Department of Public Health, University of Massachusetts Lowell, Lowell, Massachusetts; §Department of Population Health Sciences, University of Utah School of Medicine, Salt Lake City, Utah; IInstitute for Health Research, Kaiser Permanente Colorado, Aurora, Colorado; "Section of Gerontology and Geriatric Medicine, Department of Internal Medicine, Wake Forest School of Medicine, Winston-Salem, North Carolina; * Division of Nephrology and Hypertension, University of Utah School of Medicine, Salt Lake City, Utah; ${ }^{\dagger+}$ Clinical Applications and Prevention Branch, National

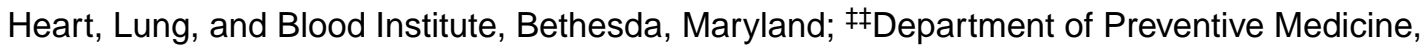
University of Tennessee Health Science Center, Memphis, Tennessee; §§Section on Cardiovascular Medicine, Department of Internal Medicine, Wake Forest School of Medicine, Winston-Salem, North Carolina; "TCardiovascular Institute, Rutgers Robert Wood Johnson Medical School, New Brunswick, New Jersey; "II'Department of Neurology, Case Western Reserve University School of Medicine, Cleveland, Ohio; ${ }^{* *}$ Department of Epidemiology, University of Alabama at Birmingham, Birmingham, Alabama; ${ }^{\dagger+}$ Department of Medicine, University of

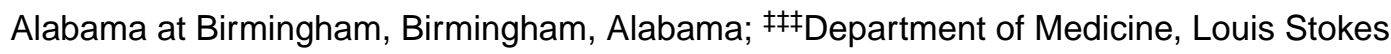

\footnotetext{
Address correspondence to Nicholas M. Pajewski, PhD, Department of Biostatistics and Data Science, Division of Public Health Sciences, Center for Health Care Innovation, Medical Center Boulevard, Winston-Salem, NC 27157. npajewsk@wakehealth.edu, Twitter handle: @NMPajewski.

Author Contributions: All listed authors contributed to the study concept and design, acquisition of participants and/or data, interpretation of data, and preparation of the manuscript. Nicholas Pajewski had full access to all the data in the study and takes responsibility for the integrity of the data and the accuracy of the data analysis.

Conflicts of Interest: All authors received support for this work from the NIH. Adam P. Bress receives research support to his institution from Novartis, Amgen, and Amarin. Jordan King has received private consulting income from Novartis and Amarin. Suzanne Oparil reported receipt of personal fees from Actelion Clinical Research, Boehringer Ingelheim/Lilly, Lundbeck, Novo Nordisk, 98point6, George Clinical Pty, Idorsia Pharmaceuticals, Pfizer, and ROX Medical, and grant support from Actelion Clinical Research, George Clinical Pty, Idorsia Pharmaceuticals, and Novartis. She also reported work as editor in chief of Current Hypertension Reports (until December 2020), published by Springer Science Business Media, for which she receives an annual stipend of \$5000. JeffD. Williamson reported that his institution received funding from Biogen (unrelated to this study).

SUPPORTING INFORMATION

Additional Supporting Information may be found in the online version of this article.
} 
Cleveland Veterans Affairs Medical Center, Case Western Reserve University, Cleveland, Ohio; $\S \S \S$ Section of Nephrology, Department of Internal Medicine, Wake Forest School of Medicine, Winston-Salem, North Carolina; ПTाDivision of Cardiovascular Sciences, National Heart, Lung, and Blood Institute, Bethesda, Maryland; "IIII'Frances Payne Bolton School of Nursing, Case Western Reserve University, Cleveland, Ohio; ${ }^{* \star *}$ Division of Geriatrics, University of Utah School of Medicine, Salt Lake City, Utah; ${ }^{\dagger+t \dagger G e r i a t r i c ~ R e s e a r c h, ~ E d u c a t i o n, ~ a n d ~ C l i n i c a l ~ C e n t e r, ~}$ Veterans Affairs Salt Lake City Health Care System, Salt Lake City, Utah; \#¥¥‡Department of Epidemiology, Tulane University School of Public Health and Tropical Medicine, New Orleans, Louisiana; §§§§Division of Nephrology and Hypertension, Department of Medicine, Case Western Reserve University, Cleveland, Ohio.

\section{Abstract}

OBJECTIVES: To evaluate the effect of intensive systolic blood pressure (SBP) control in older adults with hypertension, considering cognitive and physical function.

DESIGN: Secondary analysis.

SETTING: Systolic Blood Pressure Intervention Trial (SPRINT) PARTICIPANTS: Adults 80 years or older.

INTERVENTION: Participants with hypertension but without diabetes $(\mathrm{N}=1167)$ were randomized to an SBP target below $120 \mathrm{~mm} \mathrm{Hg}$ (intensive treatment) vs a target below $140 \mathrm{~mm}$ $\mathrm{Hg}$ (standard treatment).

MEASUREMENTS: We measured the incidence of cardiovascular disease (CVD), mortality, changes in renal function, mild cognitive impairment (MCI), probable dementia, and serious adverse events. Gait speed was assessed via a 4-m walk test, and the Montreal Cognitive Assessment (MoCA) was used to quantify baseline cognitive function.

RESULTS: Intensive treatment led to significant reductions in cardiovascular events (hazard ratio $[\mathrm{HR}]=.66 ; 95 \%$ confidence interval $[\mathrm{CI}]=.49-.90)$, mortality $(\mathrm{HR}=.67 ; 95 \% \mathrm{CI}=.48-.93)$, and MCI $(\mathrm{HR}=.70 ; 95 \% \mathrm{CI}=.51-.96)$. There was a significant interaction $(\mathrm{P}<.001)$ whereby participants with higher baseline scores on the MoCA derived strong benefit from intensive treatment for a composite of CVD and mortality ( $\mathrm{HR}=.40 ; 95 \% \mathrm{CI}=.28-.57)$, with no appreciable benefit in participants with lower scores on the MoCA $(\mathrm{HR}=1.33=95 \% \mathrm{CI}=.87-$ 2.03). There was no evidence of heterogeneity of treatment effects with respect to gait speed. Rates of acute kidney injury and declines of at least $30 \%$ in estimated glomerular filtration rate were increased in the intensive treatment group with no between group differences in the rate of injurious falls.

CONCLUSION: In adults aged 80 years or older, intensive SBP control lowers the risk of major cardiovascular events, MCI, and death, with increased risk of changes to kidney function. The cardiovascular and mortality benefits of intensive SBP control may not extend to older adults with lower baseline cognitive function.

TRIAL REGISTRATION: Clinicaltrials.gov identifier: . 


\section{Keywords}

cardiovascular disease; hypertension; older adults; cognitive function

The number of adults aged 80 years and older is steadily increasing and expected to reach $7.7 \%$ of the population in the United States by $2050 .{ }^{1}$ Given that the lifetime risk of developing hypertension is at least $70 \%$ by age 80 for whites and blacks in the United States,

2 this demographic shift will induce a growing impact of hypertension and its adverse consequences in older adults. The 2017 American College of Cardiology/American Heart Association blood pressure guidelines recommended treatment to a systolic blood pressure (SBP) below $130 \mathrm{~mm} \mathrm{Hg}$ in noninstitutionalized ambulatory community-dwelling adults 65 years of age or older. ${ }^{3}$ However, hypertension treatment for adults 80 years or older is frequently complicated by multiple chronic conditions such as frailty, polypharmacy, and cognitive impairment. ${ }^{4,5}$ Observational analyses indicate an attenuation of the association between elevated blood pressure (BP) and the incidence of vascular and non-vascular disease with increasing age, ${ }^{6,7}$ suggesting that the balance of risk to benefit for hypertension treatment may be different for adults 80 years of age or older as compared with adults in their 60s and 70s. Several studies also suggest that older adults with robust functional status may be more likely to benefit from hypertensive therapy, with weaker or null associations between elevated SBP and adverse outcomes in adults with impaired function. ${ }^{8-10}$

Much of the evidence for the benefit of antihypertensive drug therapy in adults 80 years of age or older comes from the Hypertension in the Very Elderly Trial (HYVET) which identified a significant and clinically important reduction in stroke and mortality with the long-acting diuretic indapamide (alone or combined with perindopril) compared with placebo. ${ }^{11}$ However, the baseline SBP in HYVET was $160 \mathrm{~mm} \mathrm{Hg}$ or higher, with participants assigned to indapamide achieving a mean (seated) SBP of $143.5 \mathrm{~mm} \mathrm{Hg}$ after 2 years of treatment. Thus HYVET provides limited information concerning more intensive treatment of SBP to levels below $140 \mathrm{~mm}$ Hg. The Systolic Blood Pressure Intervention Trial (SPRINT) compared treatment to an SBP goal below $120 \mathrm{~mm} \mathrm{Hg}$ (intensive treatment) with treatment to a goal of below $140 \mathrm{~mm} \mathrm{Hg}$ (standard treatment) in older adults with hypertension. ${ }^{12}$ SPRINT included a large number of participants 75 years or older, with results in this subgroup largely indicating beneficial effects on cardiovascular morbidity and mortality. ${ }^{13}$ However, most participants in this age group (55.7\%) were between 75 and 80 years of age, and very little was reported specifically for the oldest participants in SPRINT.

${ }^{14}$ Here we comprehensively examine a range of outcomes including cardiovascular morbidity and mortality, renal function, adjudicated mild cognitive impairment (MCI) and probable dementia, health-related quality of life (HRQOL), and serious adverse events. We also explore whether baseline impairments in cognitive or physical function modify the effect of intensive BP control on outcomes.

\section{METHODS}

The trial design, methods, protocol, and primary results were published previously. ${ }^{12,13,15}$ Briefly, SPRINT was a multicenter randomized clinical trial comparing two thresholds for 
managing SBP in older adults with hypertension who were at increased risk of cardiovascular disease (CVD). All participants 80 years or older were considered at increased risk for CVD by virtue of their age. Exclusion criteria included residence in a nursing home, diagnosis of dementia or use of medications for dementia therapy, prevalent diabetes, or a history of stroke. Participants were randomized to either an SBP goal of below $120 \mathrm{~mm} \mathrm{Hg}$ (intensive treatment) or below $140 \mathrm{~mm} \mathrm{Hg}$ (standard treatment), with the randomization stratified by clinic site. The study was approved by an institutional review board at each participating site, and each participant provided written informed consent.

\section{Outcomes}

Two separate committees, unaware of treatment assignment and using formal criteria and operations manuals, adjudicated protocol-specified clinical outcomes related to (1) CVD morbidity and mortality, and (2) MCI and probable dementia. The primary CVD outcome was a composite of nonfatal myocardial infarction, acute coronary syndrome not resulting in a myocardial infarction, nonfatal stroke, nonfatal acute decompensated heart failure, and death from cardiovascular causes. Secondary outcomes included all-cause mortality and the composite of the primary CVD outcome and all-cause mortality. Ascertainment of cognitive outcomes was previously described. ${ }^{16}$ Cognitive outcomes included the occurrence of probable dementia, MCI, and a composite outcome of probable dementia or MCI.

\section{Serious Adverse Events}

Serious adverse events (SAEs) were defined as events that were fatal or life threatening, resulted in significant or persistent disability, required hospitalization or resulted in prolonged hospitalization, or medical events that the investigator judged to be a significant hazard or harm to the participant and required medical or surgical intervention to prevent harm. The following conditions of interest were reported as adverse events if they were evaluated in an emergency department: hypotension, syncope, injurious falls, electrolyte abnormalities, and bradycardia. Episodes of acute kidney injury (or acute renal failure) were monitored if they led to hospitalization and were reported in the hospital discharge summary.

\section{Duration of Follow-Up}

Recruitment for the overall trial began on November 8, 2010. The director of the National Heart, Lung, and Blood Institute accepted the Data Safety and Monitoring Board recommendation to stop the intervention on August 20, 2015. Clinical outcomes in this report (with the exception of cognition) are based on additional follow-up including study "closeout" visits through July 1, 2016. During this time frame, the trial was still providing medication at no cost to participants; however, BP management decisions were gradually returned to participants' primary care physicians. For cognitive outcomes, follow-up also included an extended follow-up visit, conducted between October 2017 and July 2018.

\section{Study Measurements}

Sociodemographic data were collected at baseline, with race or ethnicity information collected via self-report. Body mass index was calculated as weight in kilograms divided by height in meters squared. The estimated glomerular filtration rate (eGFR) was calculated by 
the four-variable Modification of Diet in Renal Disease study equation. ${ }^{17}$ Comorbidity was defined based on the index of Selim et al. ${ }^{18} \mathrm{BP}$ at all study visits was determined using the mean of three properly sized automated cuff readings, taken 1 minute apart after 5 minutes of quiet rest. ${ }^{19}$

Gait speed was measured at baseline via a timed 4-m walk, performed twice at the participant's usual pace from a standing start. ${ }^{20}$ The use of a walking assistive device was permitted if typically used by the participant to walk short distances. The faster of the two gait speeds in meters per second was used in this analysis. Gait speeds slower than $.20 \mathrm{~m} / \mathrm{s}$ and faster than $2.0 \mathrm{~m} / \mathrm{s}$ were set to missing.

Patient-reported outcomes assessed annually included the Veterans RAND 12-Item Health Survey (VR-12) that describes physical and mental HRQOL. ${ }^{21}$ Scores on the Physical Component Summary (PCS) and Mental Component Summary (MCS) of the VR-12 are standardized with a mean of 50 and a standard deviation (SD) of 10; scores range from 0 to 100, with higher scores denoting better physical health and mental health, respectively.

\section{Exploratory Analyses of Cognitive and Physical Function}

We examined the effect of decrements in cognitive or physical function based on gait speed and scores on the Montreal Cognitive Assessment (MoCA). ${ }^{22}$ Participants were categorized as having lower physical function if their gait speed was slower than $.8 \mathrm{~m} / \mathrm{s}^{23}$ Lower cognitive function was defined as scoring 18 or lower (less than high school education) or 20 or lower (high school education or higher) on the MoCA. This roughly corresponds to the estimated normative 25th percentile at 80 years of age in the Irish Longitudinal Study of Aging. ${ }^{24}$

\section{Statistical Analysis}

All analyses were based on intention to treat. The time to occurrence of the primary cardiovascular outcome, MCI, probable dementia, and incident SAEs was compared using the subdistribution hazard model of Fine and Gray accounting for the competing risk of death (noncardiovascular death for the primary CVD outcome). ${ }^{25}$ All-cause mortality and composite outcomes including all-cause mortality were compared between treatment groups using Cox proportional hazards regression. For both modeling approaches, the baseline hazard function was stratified by clinical site. ${ }^{26}$ We used linear mixed-effect models to compare longitudinal trajectories for BP and HRQOL between the treatment groups. The models included random effects for participants and clinic site to account for longitudinal assessments and correlations between participants at the same clinic site. For the HRQOL measures, effect estimates are expressed as an annual slope, assuming linear change over time at the group level. We included time by randomization group interaction terms to test whether the changes in each of the longitudinal outcomes differed between the treatment groups. All hypothesis tests were meant to be hypothesis generating and conducted at an a level of .05. Because we report 40 hypothesis tests (considering multiple subgroups and a range of outcomes), there is an $87 \%$ chance that at least one test would be significant at the .05 level assuming independence between tests. All analyses were performed using SAS software v.9.4 and the R Statistical Computing Environment. 


\section{RESULTS}

Baseline characteristics of the 1167 randomized participants 80 years or older are shown in Table 1. The mean age was $83.5 \pm 3.2$ years (SD), with $3.3 \%$ participants older than 90 years at baseline. Most of the participants were male $(61.2 \%)$, white $(76.0 \%)$, with a mean systolic BP of $142.6 \pm 16.1 \mathrm{~mm} \mathrm{Hg}$. Most (89.8\%) had at least three comorbid conditions, $54.7 \%$ were taking at least five medications, and $27.2 \%$ had a history of CVD. The mean gait speed was $.87 \pm .23 \mathrm{~m} / \mathrm{s}$, with $409(36.5 \%)$ participants having a gait speed shower than $.8 \mathrm{~m} / \mathrm{s}$. The median MoCA score was 22, with 413 (35.8\%) participants scoring below the education-specific normative 25 th percentiles.

\section{Blood Pressure and Medication Use during Follow-Up}

SBP over the course of follow-up is shown in Figure 1. During the intervention phase of the trial, mean SBP averaged $123.9 \mathrm{~mm} \mathrm{Hg}$ and $135.3 \mathrm{~mm} \mathrm{Hg}$ in the intensive and standard treatment groups, respectively, for a mean difference of $11.5 \mathrm{~mm} \mathrm{Hg}$ (95\% confidence interval $[\mathrm{CI}]=10.6-12.4 \mathrm{~mm} \mathrm{Hg}$; Supplementary Table S1). Among those in the intensive treatment group, the proportion taking three or more classes of antihypertensive medications was $29.5 \%$ at baseline and increased to $48.6 \%$ at the 1 -year follow-up. Conversely, the proportion taking three or more classes decreased in the standard treatment group, moving from $31.4 \%$ to $25.0 \%$. During the extended follow-up visits, the between-group difference in SBP was attenuated to $5.6 \mathrm{~mm} \mathrm{Hg}(95 \% \mathrm{CI}=2.7-8.6 \mathrm{~mm} \mathrm{Hg})$, primarily due to an increase in the mean SBP to $130.7 \mathrm{~mm} \mathrm{Hg}$ in the intensive treatment group. The between-group difference in mean SBP did not appreciably differ by MoCA score or gait speed (Supplementary Table S1).

Although a robust between-group SBP difference was achieved, participants in the intensive treatment group tended not to have SBPs consistently below the target of $120 \mathrm{~mm} \mathrm{Hg}$. From the 6-month study visit to the end of the interventional phase of the trial, $303(54.7 \%)$ participants in the intensive treatment group achieved at least $50 \%$ of their SBP readings below $120 \mathrm{~mm} \mathrm{Hg}$, with 96 (17.3\%) participants achieving at least 80\% of their SBP readings below $120 \mathrm{~mm} \mathrm{Hg}$ (Supplementary Table S2). Participants in the intensive treatment group were more consistently controlled below $130 \mathrm{~mm} \mathrm{Hg} ; 437$ (78.9\%) and 252 (45.9\%) achieved $50 \%$ and $80 \%$ of their SBP readings below $130 \mathrm{~mm} \mathrm{Hg}$, respectively.

\section{Clinical Outcomes}

In the intensive treatment group, 75 participants experienced a primary composite CVD event compared with 106 participants in the standard treatment group (hazard ratio [HR] $=.67 ; 95 \% \mathrm{CI}=.50-.90)$ (Table 2). Participants in the intensive treatment group also experienced a reduction in all-cause mortality $(\mathrm{HR}=.67 ; 95 \% \mathrm{CI}=.49-.92)$. For both incident CVD events and mortality, significant interactions were found between subgroups based on MoCA score and treatment group. For example, a strongly beneficial effect of intensive treatment on all-cause mortality was found $(\mathrm{HR}=.39 ; 95 \% \mathrm{CI}=.24-.64)$ for participants with MoCA scores above the normative 25th percentiles. In contrast, the rate of all-cause mortality was numerically higher in participants scoring at or below the normative 25th percentiles randomized to intensive treatment $(\mathrm{HR}=1.19$; 95\% $\mathrm{CI}=.72-1.97$; 
interaction $\mathrm{P}$ value $=.003)$. There was an increased risk of experiencing a $30 \%$ reduction in eGFR ( $\mathrm{HR}=3.41 ; 95 \% \mathrm{CI}=1.92-6.06)$ with intensive treatment, with the relative effect largely independent of cognitive and physical function. The incidence of probable dementia was similar between the treatment groups; however, participants in the intensive treatment group had a lower risk of MCI $(\mathrm{HR}=.72 ; 95 \% \mathrm{CI}=.53-.98$; Table 3$)$. No evidence of heterogeneity for the cognitive outcomes by either MoCA score or gait speed was observed.

\section{Serious Adverse Events}

In the intensive treatment group, SAEs occurred in 340 participants compared with 353 participants in the standard treatment group ( $\mathrm{HR}=.92 ; 95 \% \mathrm{CI}=.79-1.07$; Table 4). Rates of acute kidney injury or renal failure ( $\mathrm{HR}=2.12 ; 95 \% \mathrm{CI}=1.37-3.26)$ were increased in the intensive treatment group; however, no difference was observed in the incidence of injurious falls ( $\mathrm{HR}=.93 ; 95 \% \mathrm{CI}=.64-1.36)$. There were no significant interactions for SAEs by gait speed (Supplementary Table S3). Participants in the intensive treatment group had a higher rate of laboratory alerts for serum sodium values below $130 \mathrm{mmol} / \mathrm{L}(\mathrm{HR}=$ $1.78 ; 95 \%$ CI $=1.03-3.05$; Supplementary Table S4).

\section{Health-Related Quality of Life}

No differences were found between the intensive and standard treatment groups in mental quality of life based on the VR-12 MCS score, either overall or by MoCA score or gait speed (Supplementary Table S5). A smaller rate of decline was observed in the PCS score for participants in the intensive treatment group (mean difference $=.33 ; 95 \% \mathrm{CI}=.06-.60 ; \mathrm{P}$ $=.02$ ). This roughly corresponds to a mean difference of slightly more than 1 point over 4 years, which is generally thought to be a small effect. ${ }^{27}$

\section{DISCUSSION}

Participants 80 years or older randomized to an intensive SBP target of below $120 \mathrm{~mm} \mathrm{Hg}$ as compared with a target of below $140 \mathrm{~mm} \mathrm{Hg}$ experienced a decreased risk of cardiovascular morbidity and mortality, MCI, and all-cause mortality. These effects were accompanied by an increased risk of declines in renal function and an increased risk of acute kidney injury but not an increased risk of injurious falls. In general, these results point to a favorable risk benefit profile for intensive BP control in adults 80 years or older, given other work from SPRINT indicating that most cases of acute kidney injury were transient, eventually leading to recovery of kidney function. ${ }^{28}$ In addition, the lack of increased risk of injurious falls is especially critical given recent statistics indicating an increasing mortality rate due to falls in adults 75 years or older and the concern that hypotension can result in falls. ${ }^{29}$

Analyses of the Systolic Hypertension in the Elderly Program suggested differential effects of hypertension treatment based on the presence or absence of physical activity limitations. ${ }^{9}$ Here we did not observe differential treatment effects with respect to gait speed but did find rather striking differences with respect to cognitive function. Participants with higher baseline cognitive function ( $>60 \%$ of participants $>00 \mathrm{y}$ ) derived a strong benefit from intensive SBP control with respect to CVD and mortality, whereas participants with lower cognitive function randomized to intensive SBP control experienced numerically higher 
rates of CVD and mortality. Note that a similar pattern of effect also holds with respect to CVD and mortality among the larger set of participants 75 years or older in SPRINT (Supplementary Table S6). This result was somewhat unexpected given previous analyses from HYVET $^{30}$ and SPRINT ${ }^{13}$ that examined heterogeneity through the use of frailty indices that incorporate measures of cognitive and physical function. Those analyses did not suggest any significant heterogeneity in the intervention effect in either trial by frailty status, despite a clear gradient of risk whereby participants with higher frailty index scores experienced higher rates of CVD and mortality.

Although our results add support to considering cognitive function in clinical decision making for hypertension therapy, ${ }^{31}$ the precise threshold at which inaction or even deprescribing should be preferred is unclear. ${ }^{5}$ One limitation of SPRINT's design is that the presence of MCI was not adjudicated at baseline, and so we were forced to use an ad hoc categorization of cognitive status based on the MoCA. Although we categorized participants based on age and education-specific normative data, the thresholds we used were derived from an Irish population and have not been validated. Focusing on a general cognitive screening instrument like the MoCA has advantages in terms of clinical implementation, but there are numerous barriers to routine objective measurement of cognitive function.

Although the Medicare Annual Wellness Visits provide an appealing context for ascertaining function, they are underused ${ }^{32}$ and may not adequately detect impairment with respect to cognition. ${ }^{33}$ In addition, there are a number of recent concerns with the MoCA specifically related to monetization. ${ }^{34}$

Moving beyond function, several other aspects of these results should be considered. First, most SPRINT participants 80 years or older had ages that were clustered between 80 and 85 years, with only $24.9 \%$ and $3.3 \%$ (of those $\$ 80$ y) older than 85 and 90 years of age at the time of randomization. Therefore, the SPRINT results are most informative for adults 85 years of age or younger, and they are less relevant for intensification of antihypertensive therapy in adults older than 85 years. A second consideration is that participants 80 years or older randomized to intensive SBP control averaged a mean SBP of $125 \mathrm{~mm} \mathrm{Hg}$ during the interventional phase of the trial, with less than $10 \%$ consistently controlled to below $120 \mathrm{~mm}$ $\mathrm{Hg}$, despite the treatment target of below $120 \mathrm{~mm} \mathrm{Hg}$ and systematic measurement of BP. ${ }^{19}$ It is clear that achieved SBP with more intensive hypertensive therapy will tend to be higher in this age range as compared with younger adults. This difference needs to be considered in light of differences between the automated protocol-based BP measurement procedure used in SPRINT and what is typically done in clinical practice. ${ }^{35}$ Finally, given that this is a secondary analysis, our results should be interpreted with caution, also recognizing more general limitations of SPRINT including generalizability of the cohort and early cessation of the trial intervention. ${ }^{36,37}$

In conclusion, in adults aged 80 years or older, intensive SBP control lowers the risk of major cardiovascular events, MCI, and death, with increased risk of changes to kidney function. The cardiovascular and mortality benefits of intensive SBP control may not extend to older adults with lower cognitive function. 


\section{Supplementary Material}

Refer to Web version on PubMed Central for supplementary material.

\section{ACKNOWLEDGMENTS}

Financial Disclosure: The Systolic Blood Pressure Intervention Trial was funded by the National Institutes of Health (NIH) (including the National Heart, Lung, and Blood Institute [NHLBI], the National Institute of Diabetes and Digestive and Kidney Diseases, the National Institute on Aging, and the National Institute of Neurological Disorders and Stroke) under contracts HHSN268200900040C, HHSN268200900046C, HHSN268200900047C, HHSN268200900048C, and HHSN 268200900049C and interagency agreement A-HL-13-002-001. It was also supported in part with resources and use of facilities through the Department of Veterans Affairs. Azilsartan and chlorthalidone (combined with azilsartan) were provided by Takeda Pharmaceuticals International.

Additional support was provided through the following National Center for Advancing Translational Sciences clinical and translational science awards: UL1TR000439 (awarded to Case Western Reserve University); UL1RR025755 (Ohio State University); UL1RR024134 and UL1TR000003 (University of Pennsylvania); UL1RR025771 (Boston University); UL1TR000093 (Stanford University); UL1RR025752, UL1TR000073, and UL1TR001064 (Tufts University); UL1TR000050 (University of Illinois); UL1TR000005 (University of Pittsburgh); U54TR000017-06 (University of Texas Southwestern Medical Center); UL1TR000105-05 (University of Utah); UL1 TR000445 (Vanderbilt University); UL1TR000075 (George Washington University); UL1 TR000002 (University of California, Davis); UL1 TR000064 (University of Florida); and UL1TR000433 (University of Michigan); and by National Institute of General Medical Sciences, Centers of Biomedical Research Excellence award NIGMS P30GM103337 (awarded to Tulane University). Additional support was also provided by R01AG055606, K01HL133468 (Adam P. Bress), the Wake Forest Claude Pepper Center (P30AG021332), and the Alzheimer's Association.

The views expressed here are those of the authors and do not represent the official position of the NIH, the NHLBI, the Department of Veterans Affairs, the US government, or the SPRINT Research Group. This article was not reviewed by the SPRINT Publications and Presentations Committee.

Sponsor's Role: The SPRINT steering committee designed and conducted the study including the collection and management of the data. Scientists at the National Institutes of Health (NIH; the sponsor) as a group and the principal investigator of the Veterans Affairs clinical network had one vote on the steering committee of the trial that had seven voting members. The NIH and the US Department of Veterans Affairs had roles in the design and conduct of the study; collection, management, analysis, and interpretation of the data; and preparation, review, and approval of the manuscript but not in the decision to submit the manuscript for publication.

\section{REFERENCES}

1. Ortman JM, Velkoff VA, Hogan H. An Aging Nation: The Older Population in the United States. Current Population Reports. U.S. Census Bureau; 2014 https://www.census.gov/prod/2014pubs/ p25-1140.pdf. Accessed July 16, 2019.

2. Chen V, Ning H, Allen N, et al. Lifetime risks for hypertension by contemporary guidelines in African American and white men and women. JAMA Cardiol. 2019;4(5):455-459. [PubMed: 30916719]

3. Whelton PK, Carey RM, Aronow WS, et al. 2017 ACC/AHA/AAPA/ABC/ACPM/AGS/ APhA/ASH/ASPC/NMA/PCNA Guideline for the Prevention, Detection, Evaluation, and Management of High Blood Pressure in Adults: Executive Summary: a Report of the American College of Cardiology/American Heart Association Task Force on Clinical Practice Guidelines. Circulation. 2018;138(17): e426-e483. [PubMed: 30354655]

4. Forman DE, Maurer MS, Boyd C, et al. Multimorbidity in older adults with cardiovascular disease. J Am Coll Cardiol. 2018;71(19):2149-2161. [PubMed: 29747836]

5. Benetos A, Petrovic M, Strandberg T. Hypertension management in older and frail older patients. Circ Res. 2019;124(7):1045-1060. [PubMed: 30920928]

6. Rapsomaniki E, Timmis A, George J, et al. Blood pressure and incidence of twelve cardiovascular diseases: lifetime risks, healthy life-years lost, and age-specific associations in 125 million people. Lancet. 2014;383(9932):1899-1911. [PubMed: 24881994] 
7. Lacey B, Lewington S, Clarke R, et al. Age-specific association between blood pressure and vascular and non-vascular chronic diseases in 05 million adults in China: a prospective cohort study. Lancet Glob Health. 2018;6(6): e641-e649. [PubMed: 29773120]

8. Odden MC, Peralta CA, Haan MN, Covinsky KE. Rethinking the association of high blood pressure with mortality in elderly adults: the impact of frailty. Arch Intern Med. 2012;172(15):1162-1168. [PubMed: 22801930]

9. Charlesworth CJ, Peralta CA, Odden MC. Functional status and antihypertensive therapy in older adults: a new perspective on old data. Am J Hypertens. 2016;29(6):690-695. [PubMed: 26541570]

10. Wu C, Smit E, Peralta CA, Sarathy H, Odden MC. Functional status modifies the association of blood pressure with death in elders: health and retirement study. J Am Geriatr Soc. 2017;65(7):1482-1489. [PubMed: 28306145]

11. Beckett NS, Peters R, Fletcher AE, et al. Treatment of hypertension in patients 80 years of age or older. N Engl J Med. 2008;358(18):1887-1898. [PubMed: 18378519]

12. SPRINT Research Group Wright JT, Williamson JD, et al. A randomized trial of intensive versus standard blood-pressure control. N Engl J Med. 2015;373(22):2103-2116. [PubMed: 26551272]

13. Williamson JD, Supiano MA, Applegate WB, et al. Intensive vs standard blood pressure control and cardiovascular disease outcomes in adults aged $\geq 75$ years: a randomized clinical trial. JAMA. 2016;315(24):2673-2682. [PubMed: 27195814]

14. Yaffe K. Prevention of cognitive impairment with intensive systolic blood pressure control. JAMA. 2019;321(6):548-549. [PubMed: 30688980]

15. Ambrosius WT, Sink KM, Foy CG, et al. The design and rationale of a multicenter clinical trial comparing two strategies for control of systolic blood pressure: the systolic blood pressure intervention trial (SPRINT). Clin Trials. 2014;11(5):532-546. [PubMed: 24902920]

16. SPRINT MIND Investigators for the SPRINT Research Group, Williamson JD, Pajewski NM, Auchus AP, et al. Effect of intensive vs standard blood pressure control on probable dementia: a randomized clinical trial. JAMA. 2019;321(6):553-561. [PubMed: 30688979]

17. Levey AS, Coresh J, Greene T, et al. Using standardized serum creatinine values in the modification of diet in renal disease study equation for estimating glomerular filtration rate. Ann Intern Med. 2006;145(4):247-254. [PubMed: 16908915]

18. Selim AJ, Fincke G, Ren XS, et al. Comorbidity assessments based on patient report: results from the Veterans Health Study. J Ambul Care Manage. 2004;27(3):281-295. [PubMed: 15287217]

19. Johnson KC, Whelton PK, Cushman WC, et al. Blood pressure measurement in SPRINT (Systolic Blood Pressure Intervention Trial). Hypertension. 2018; 71(5):848-857. [PubMed: 29531173]

20. Odden MC, Peralta CA, Berlowitz DR, et al. Effect of intensive blood pressure control on gait speed and mobility limitation in adults 75 years or older: a randomized clinical trial. JAMA Intern Med. 2017;177(4):500-507. [PubMed: 28166324]

21. Selim AJ, Rogers W, JA F, et al. Updated U.S. population standard for the Veterans RAND 12-item Health Survey (VR-12). Qual Life Res. 2009;18(1): 43-52. [PubMed: 19051059]

22. Nasreddine ZS, Phillips NA, Bédirian V, et al. The Montreal Cognitive Assessment, MoCA: a brief screening tool for mild cognitive impairment. J Am Geriatr Soc. 2005;53(4):695-699. [PubMed: 15817019]

23. Beaudart C, McCloskey E, Bruyère $\mathrm{O}$, et al. Sarcopenia in daily practice: assessment and management. BMC Geriatr. 2016;16(1):170. [PubMed: 27716195]

24. Kenny RA, Coen RF, Frewen J, Donoghue OA, Cronin H, Savva GM. Normative values of cognitive and physical function in older adults: findings from the Irish Longitudinal Study on Ageing. J Am Geriatr Soc. 2013;61 (Suppl 2)):S279-S290. [PubMed: 23662720]

25. Fine JP, Gray RJ. A proportional hazards model for the subdistribution of a competing risk. J Am Stat Assoc. 1999;94(446):496-509.

26. Glidden DV, Vittinghoff E. Modelling clustered survival data from multicentre clinical trials. Stat Med. 2004;23(3):369-388. [PubMed: 14748034]

27. Selim AJ, Qian SX, Rogers W, et al. Health status in adults with chronic conditions: intervention strategies for improving patient-reported outcomes. J Ambul Care Manage. 2019;42(1):2-20. [PubMed: 30499897] 
28. Rocco MV, Sink KM, Lovato LC, et al. Effects of intensive blood pressure treatment on acute kidney injury events in the systolic blood pressure intervention trial (SPRINT). Am J Kidney Dis. 2018;71(3):352-361. [PubMed: 29162340]

29. Hartholt KA, Lee R, Burns ER, van Beeck EF. Mortality from falls among US adults aged 75 years or older, 2000-2016. JAMA. 2019;321(21):2131-2133. [PubMed: 31162561]

30. Warwick J, Falaschetti E, Rockwood K, et al. No evidence that frailty modifies the positive impact of antihypertensive treatment in very elderly people: an investigation of the impact of frailty upon treatment effect in the HYpertension in the Very Elderly Trial (HYVET) study, a double-blind, placebocontrolled study of antihypertensives in people with hypertension aged 80 and over. BMC Med. 2015;13:8. [PubMed: 25592833]

31. High KP, Zieman S, Gurwitz J, et al. Use of functional assessment to define therapeutic goals and treatment. J Am Geriatr Soc. 2019;67(9):1782-1179. [PubMed: 31081938]

32. Ganguli I, Souza J, McWilliams JM, Mehrotra A. Practices caring for the underserved are less likely to adopt Medicare's annual wellness visit. Health Aff (Millwood). 2018;37(2):283-291. [PubMed: 29401035]

33. Fowler NR, Campbell NL, Pohl GM, et al. One-year effect of the Medicare annual wellness visit on detection of cognitive impairment: a cohort study. J Am Geriatr Soc. 2018;66(5):969-975. [PubMed: 29608782]

34. Borson S, Sehgal M, Chodosh J. Monetizing the MoCA: what now? J Am Geriatr Soc. 2019;67(11):2229-2231. 10.1111/jgs.16158. [PubMed: 31478562]

35. Drawz P. Clinical implications of different blood pressure measurement techniques. Curr Hypertens Rep. 2017;19(7):54. [PubMed: 28551831]

36. Supiano MA, Williamson JD. Applying the systolic blood pressure intervention trial results to older adults. J Am Geriatr Soc. 2017;65(1):16-21. [PubMed: 28111758]

37. Rich MW, Ouslander JG. Hypertension in older adults in the wake of the systolic blood pressure intervention trial. J Am Geriatr Soc. 2018;66(4): 652-654. [PubMed: 29601078] 


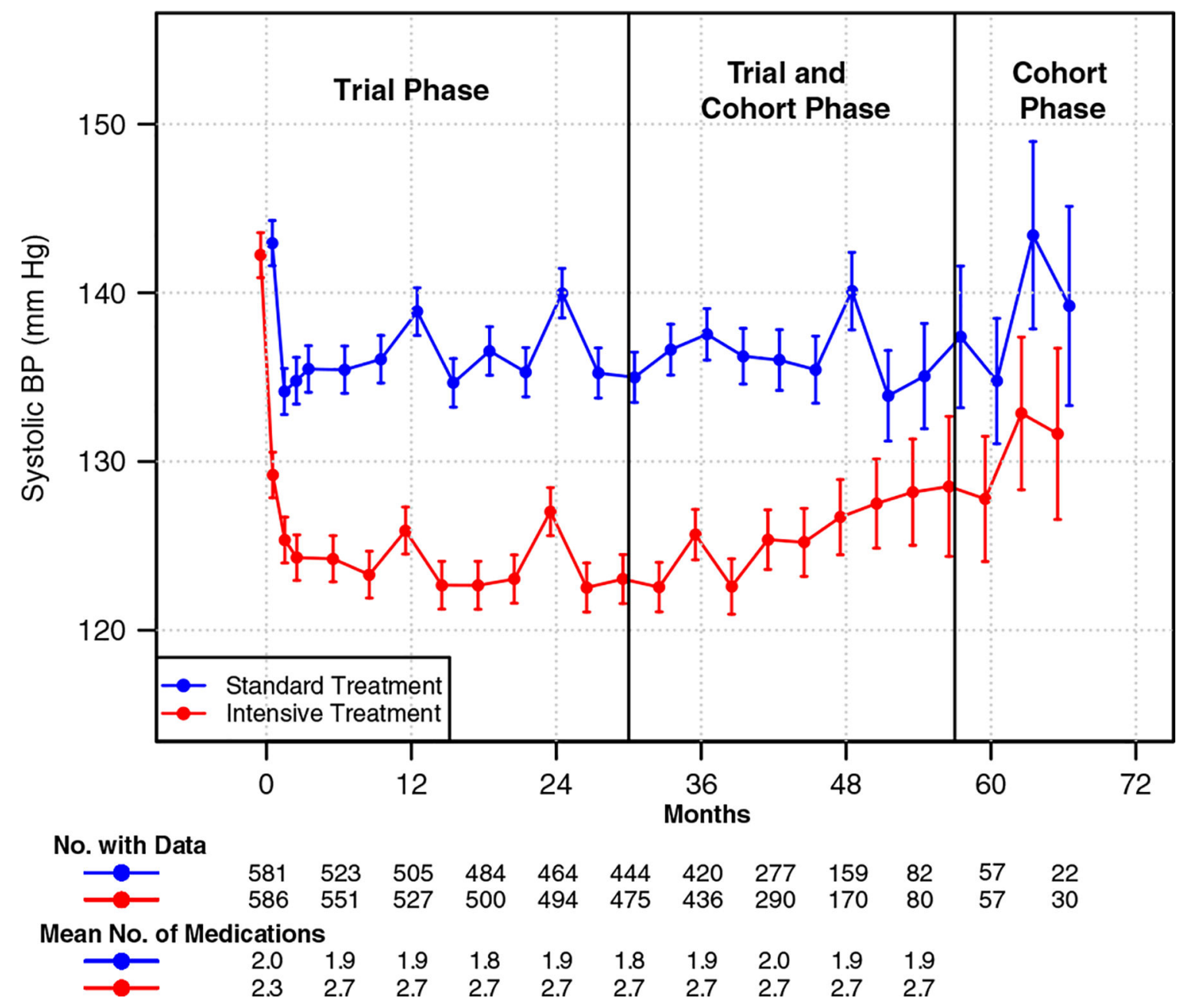

Figure 1.

Systolic blood pressure in the two treatment groups over the course of follow-up. The systolic blood pressure (SBP) target was $<120 \mathrm{~mm} \mathrm{Hg}$ in the Intensive Treatment group, and $<140 \mathrm{~mm} \mathrm{Hg}$ in the Standard Treatment group. Trial phase includes follow-up through the decision to stop the intervention on August 20, 2015; cohort phase denotes visits that occurred after that date. Points indicate least square means based on linear mixed model with error bars denoting $95 \%$ confidence intervals. 
Table 1.

Baseline Characteristics of Participants Aged 80 Years or Older

\begin{tabular}{|c|c|c|}
\hline & Intensive treatment $N=586$ & Standard treatment $\mathbf{N}=\mathbf{5 8 1}$ \\
\hline \multicolumn{3}{|l|}{ Age } \\
\hline Mean (SD), y & $83.3(3.0)$ & $83.7(3.3)$ \\
\hline$>85 \mathrm{y}, \mathrm{n}(\%)$ & $136(23.2)$ & $154(26.5)$ \\
\hline$>90 \mathrm{y}, \mathrm{n}(\%)$ & $16(2.7)$ & $22(3.8)$ \\
\hline Female sex, n (\%) & $221(37.7)$ & $231(39.8)$ \\
\hline \multicolumn{3}{|l|}{ Race/Ethnicity, n (\%) } \\
\hline White & $442(75.4)$ & 445 (76.6) \\
\hline Black & $95(16.2)$ & $93(16.0)$ \\
\hline Hispanic & $42(7.2)$ & $38(6.5)$ \\
\hline Other & $7(1.2)$ & $5(.9)$ \\
\hline Body mass index, mean (SD), kg/m² & $27.1(4.8)$ & $27.0(4.6)$ \\
\hline \multicolumn{3}{|l|}{ Seated blood pressure, mean (SD), mm Hg } \\
\hline Systolic & $142.2(15.6)$ & $142.9(16.6)$ \\
\hline Diastolic & $70.0(11.3)$ & $70.0(11.2)$ \\
\hline Orthostatic hypotension, n (\%) & $55(9.5)$ & $59(10.2)$ \\
\hline History of CVD, n (\%) & $165(28.2)$ & $152(26.2)$ \\
\hline \multicolumn{3}{|l|}{ Estimated GFR ${ }^{a}$} \\
\hline Mean (SD), $\mathrm{mL} / \mathrm{min} / 1.73 \mathrm{~m}^{2}$ & $60.9(18.3)$ & $59.6(17.8)$ \\
\hline$<60 \mathrm{~mL} / \mathrm{min} / 1.73 \mathrm{~m}^{2}, \mathrm{n}(\%)$ & $288(49.6)$ & $303(52.5)$ \\
\hline Urinary albumin to creatinine ratio, median (IQR), mg/g & $15.0(8.0-39.4)$ & $16.1(9.0-41.4)$ \\
\hline No. of medications, mean (SD) & $6.6(3.6)$ & $6.4(3.6)$ \\
\hline No. of antihypertensive agents, mean (SD) & $1.9(1.0)$ & $1.9(1.1)$ \\
\hline Statin use, $\mathrm{n}(\%)$ & $287(49.7)$ & $305(53.1)$ \\
\hline Aspirin use, $\mathrm{n}(\%)$ & $364(62.3)$ & $339(58.5)$ \\
\hline \multicolumn{3}{|l|}{ Gait speed } \\
\hline Median (IQR), m/s & $.86(.72-1.00)$ & $.87(.73-1.02)$ \\
\hline Speed $<.8 \mathrm{~m} / \mathrm{s}, \mathrm{n}(\%)$ & $204(36.2)$ & $205(36.8)$ \\
\hline MoCA score, $\mathrm{n}(\%)^{b}$ & $22(19-24)$ & $22(19-24)$ \\
\hline$\leq 18(<\mathrm{HS}) / \underline{2} 0(\Psi$HS$)$ & $208(36.0)$ & $205(35.5)$ \\
\hline VR-12 PCS, mean (SD) ${ }^{c}$ & $42.4(10.4)$ & $43.4(9.7)$ \\
\hline VR-12 MCS, mean (SD) ${ }^{\mathcal{c}}$ & $54.6(8.2)$ & $54.9(8.6)$ \\
\hline
\end{tabular}

Abbreviations: CVD, cardiovascular disease; GFR, glomerular filtration rate; HS, high school education; IQR, interquartile range; MCS, Mental Component Summary; MoCA, Montreal Cognitive Assessment; PCS, Physical Component Summary; SD, standard deviation; VR-12, Veterans RAND 12-Item Health Survey.

${ }^{a}$ Based on the four-variable Modification of Diet in Renal Disease equation.

${ }^{b}$ Scores range from 0 to 30 , with higher scores denoting better cognitive function.

${ }^{c}$ Scores on the PCS and MCS of the VR-12 are standardized with a mean of 50 and an SD of 10; scores range from 0 to 100, with higher scores denoting better physical health and mental health, respectively. 


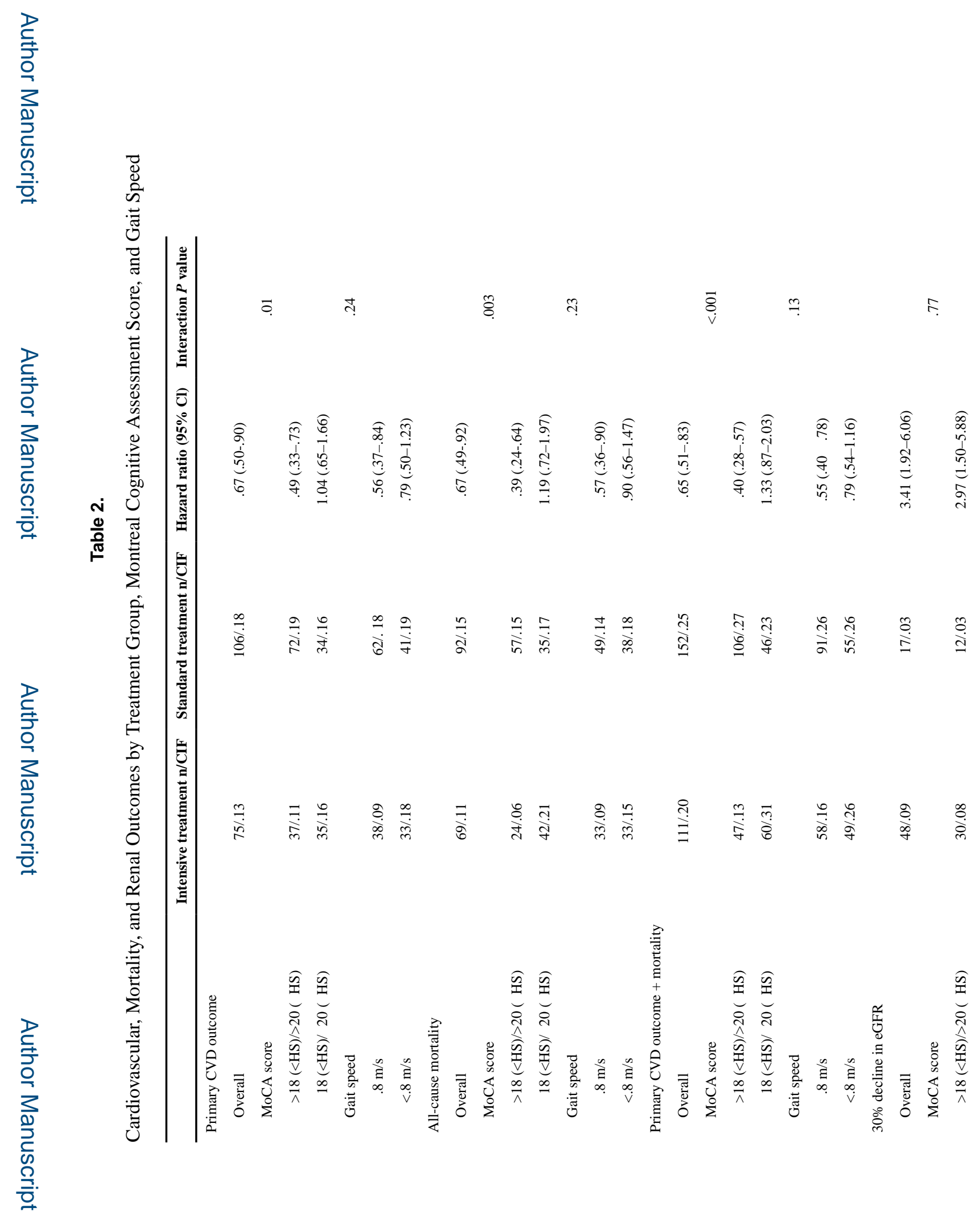




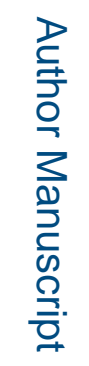

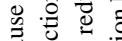

壱苛

tᄒ

औ

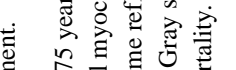

的焉

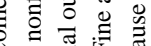

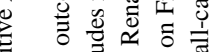

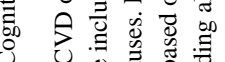

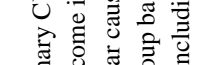

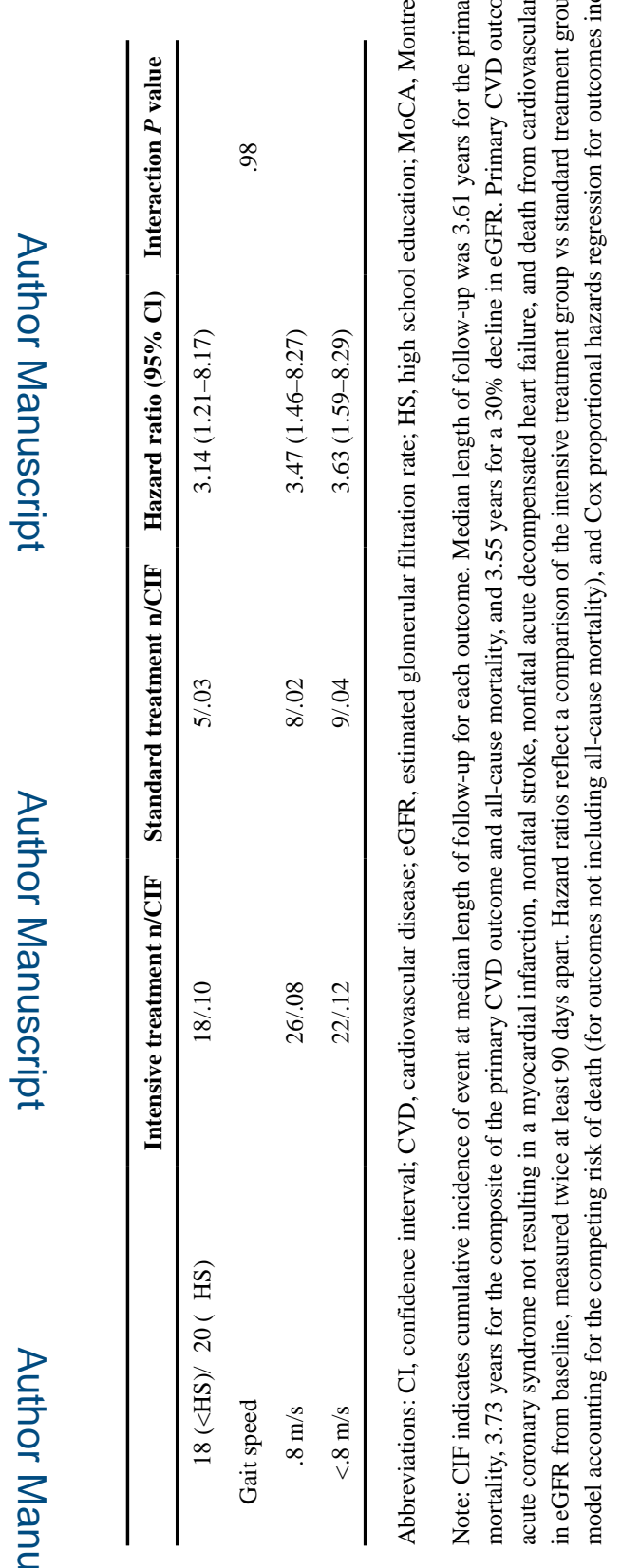

$$
\text { อ }
$$

호. 


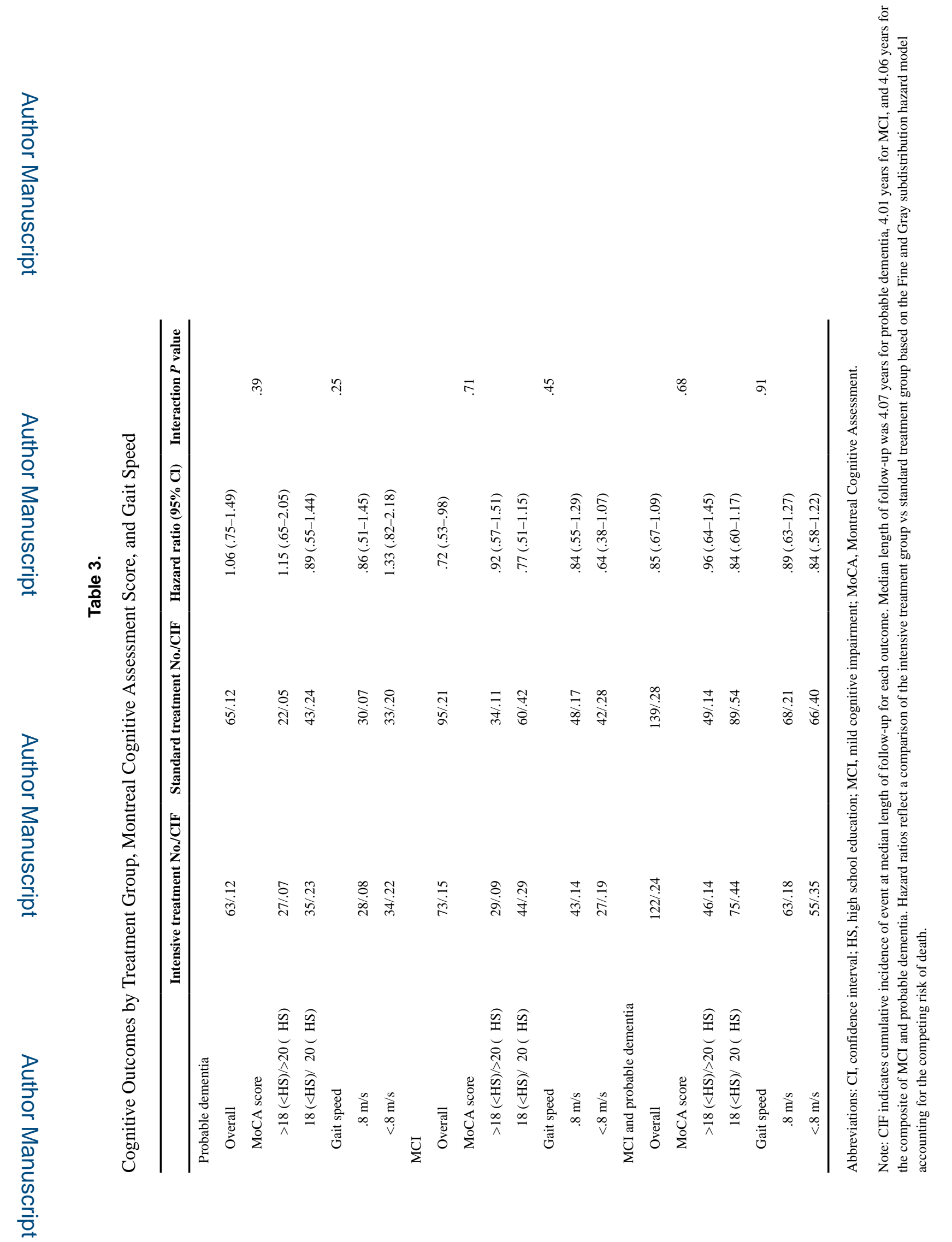




\section{롤 \\ }

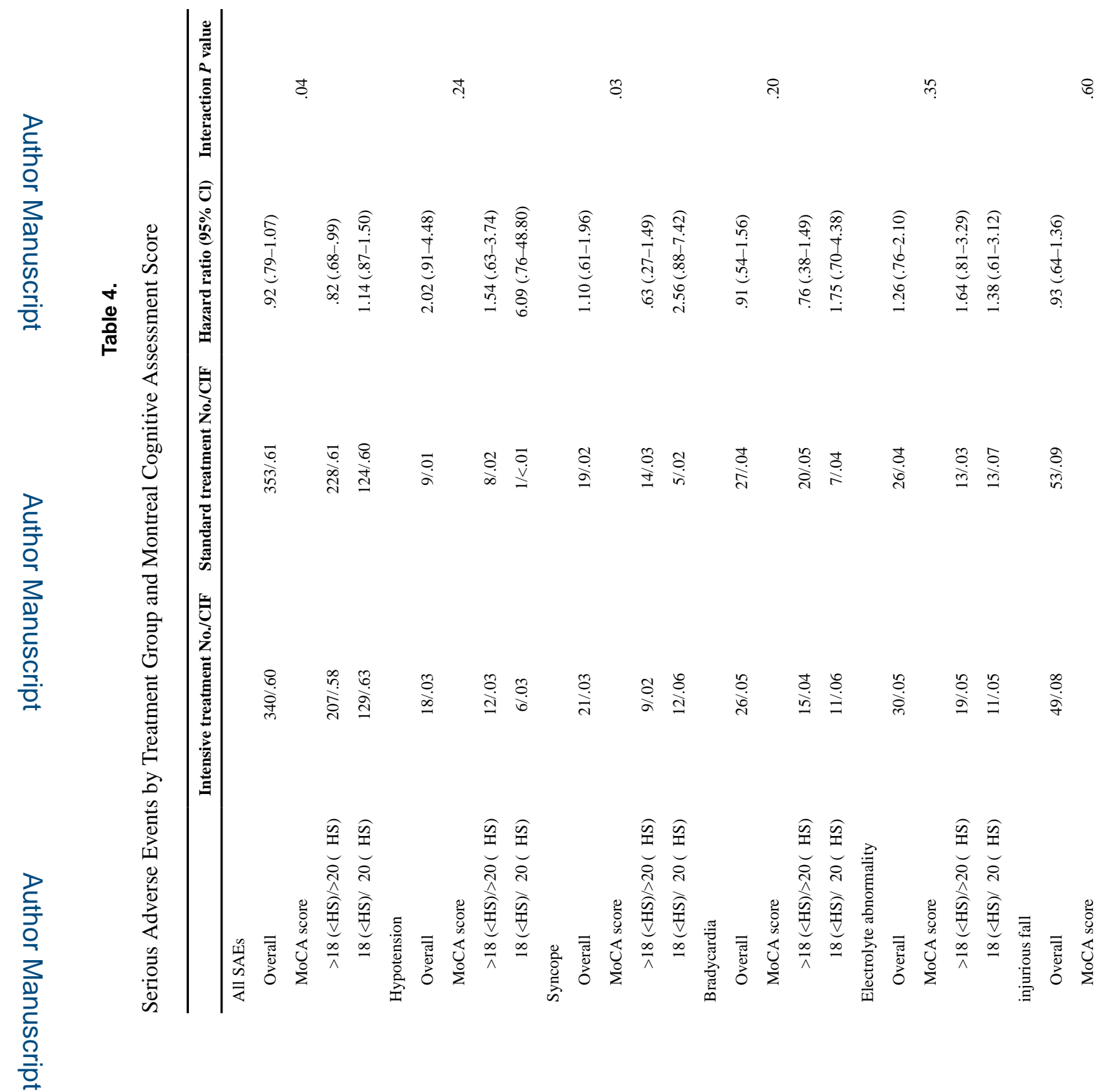

J Am Geriatr Soc. Author manuscript; available in PMC 2020 March 05. 


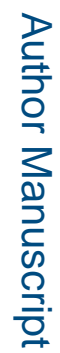

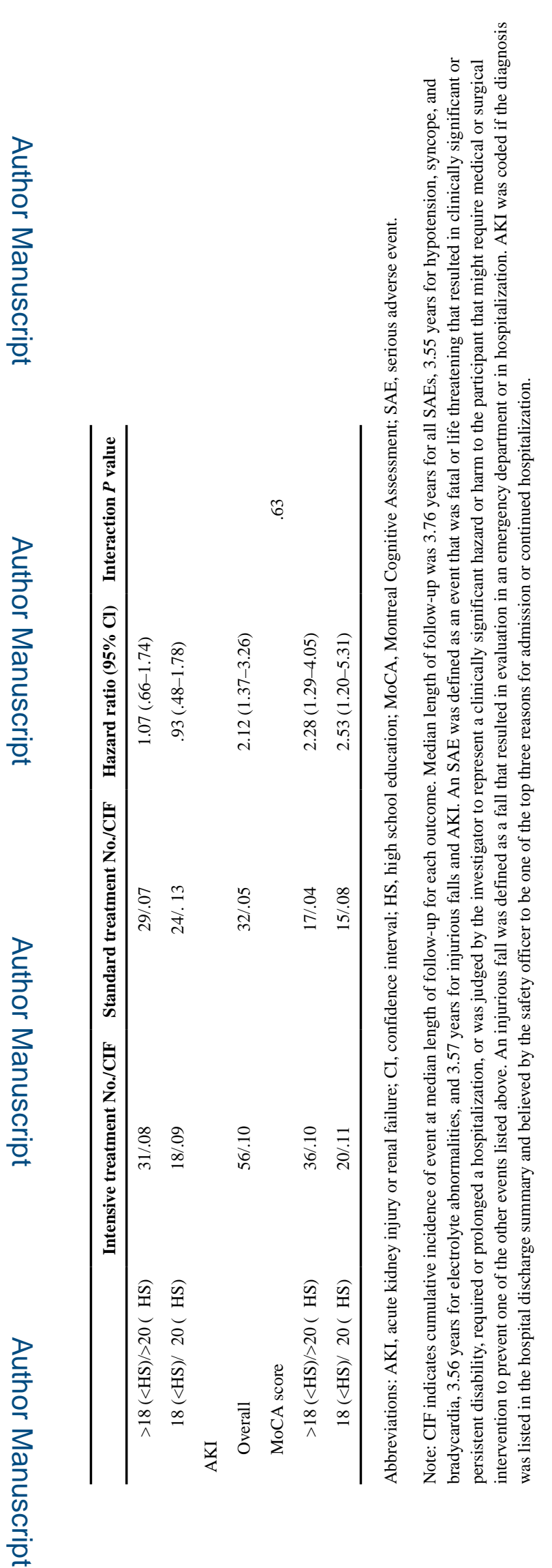

J Am Geriatr Soc. Author manuscript; available in PMC 2020 March 05. 